\title{
Renormalized stress-energy tensor of an evaporating spinning black hole
}

\author{
Adam Levi ${ }^{1}$, Ehud Eilon ${ }^{1}$, Amos Ori $^{1}$ and Maarten van de Meent ${ }^{2}$ \\ ${ }^{1}$ Department of physics, Technion-Israel Institute of Technology, Haifa 32000, Israel. \\ ${ }^{2}$ Mathematical Sciences, University of Southampton, Southampton SO17 1BJ, United Kingdom.
}

\begin{abstract}
We employ a recently developed mode-sum regularization method to compute the renormalized stress-energy tensor of a quantum field in the Kerr background metric (describing a stationary spinning black hole). More specifically, we consider a minimally-coupled massless scalar field in the Unruh vacuum state, the quantum state corresponding to an evaporating black hole. The computation is done here for the case $a=0.7 \mathrm{M}$, using two different variants of the method: $t$ splitting and $\varphi$-splitting, yielding good agreement between the two (in the domain where both are applicable). We briefly discuss possible implications of the results for computing semiclassical corrections to certain quantities, and also for simulating dynamical evaporation of a spinning black hole.
\end{abstract}

Like many great discoveries, Hawking's discovery of black-hole (BH) evaporation [1] opened a number of new profound questions. Two such outstanding questions are the information loss puzzle, and - more generally what is the end state of $\mathrm{BH}$ evaporation. A closely related problem is the detailed study of the semiclassical evaporation process. Hawking's original analysis uses quantum field theory in curved spacetime to determine the flux that a $\mathrm{BH}$ emits to infinity. However, a more detailed investigation of $\mathrm{BH}$ evaporation requires not just the outflux at infinity but also the full renormalized stress-energy tensor (RSET) $\left\langle T_{\alpha \beta}\right\rangle_{\text {ren }}$; namely, the contribution of the quantum field fluctuations to the local stress-energy tensor. It can then be inserted in the semiclassical Einstein equation

$$
G_{\alpha \beta}=8 \pi\left\langle T_{\alpha \beta}\right\rangle_{r e n}
$$

to investigate the back-reaction on the metric. Here $G_{\alpha \beta}$ is the Einstein tensor, and throughout this paper we use general-relativistic units $G=c=1$, along with $(-+++)$ signature.

The calculation of the RSET is a long-standing challenge, even for a prescribed background metric. The naive quantum-field computation yields a divergent mode sum. To renormalize it one can use the point-splitting procedure, originally developed by DeWitt [2] for $\left\langle\phi^{2}\right\rangle_{\text {ren }}$ and later adjusted to the RSET by Christensen [3]. The point-splitting scheme proves to be very useful when the field modes are known analytically. However in our case of interest - BH backgrounds - the field's modes are known only numerically, making the naive implementation of the scheme impractical. To overcome this difficulty, Candelas, Howard, Anderson and others [4 7] developed a method to implement point-splitting numerically. This method requires a fourth order WKB expansion. Since performing high-order WKB expansion is extremely difficult in Lorentzian spacetime, they used Wick rotation and carried the actual calculation in the Euclidean sector. This clever trick is very restrictive however, as the Euclidean sector does not generically exist. The most general case where this method was imple- mented is a static spherically-symmetric background [7. 36

Note also that on going to the Euclidean sector one cannot compute the RSET directly in Unruh state, as the latter is not defined there. Instead, one has to compute another state (e.g. Boulware) in the Euclidean sector, and then use the technique introduced by Elster [9] to compute the difference between two states (a non-divergent quantity) in the Lorentzian sector. This method was used to compute the RSET in Schwarzschild in Unruh state for conformally-coupled scalar field [9] and also for electromagnetic field [10].

The traditional method [4-7] is inapplicable to the Kerr geometry (describing a spinning $\mathrm{BH}$ ) which is neither spherically-symmetric nor static - and does not admit a Euclidean sector. Spinning BH solutions are considerably more realistic than static ones, because astrophysical $\mathrm{BHs}$ do generally rotate. A fascinating demonstration of this fact was recently provided by the two gravitational-wave signals discovered by LIGO: 11 In both merger events GW150914 and GW151226 the final BHs were significantly spinning (with $a / M \sim 0.7$ ).

The above discussion highlights the importance of generalizing the methods of RSET computation from spherical static BHs to the Kerr case. Over the years Ottewill, Casals, Winstanley, Duffy and others made remarkable progress by posing various quantum states on the Kerr metric [12, 13], and also by computing RSET differences between pairs of quantum states [13, 14] (which are regular). Another approach was to compute the RSET for rotating BHs in 2+1D [15, 16] as a toy model for $3+1 \mathrm{D}$.

Recently, a novel approach for implementing pointsplitting and computing $\left\langle\phi^{2}\right\rangle$ and $\left\langle T_{\alpha \beta}\right\rangle_{\text {ren }}$ in BH spacetimes was introduced by Levi and Ori [17-19, to which we shall refer here as the "Pragmatic Mode-sum Regularization" (PMR) method. This method does not resort to the Euclidean sector or to WKB expansion. Basically it only requires the background to admit a single (continuous) symmetry. PMR comes in several variants, depending on the symmetry being employed. So far two variants were introduced in detail, the $t$-splitting [17] and 
angular-splitting [18] variants, applicable to stationary or spherically-symmetric backgrounds respectively. For the sake of simplicity the presentation in 17, 18 was restricted to the regularization of $\left\langle\phi^{2}\right\rangle$, which is technically simpler. A third variant, $\varphi$-splitting (also named "azimuthal splitting"), aimed for axially-symmetric backgrounds, was also briefly introduced, in a very recent paper [19] which presented the RSET computation in Schwarzschild using PMR. All three variants were used in that paper, showing very good agreement between the three splittings.

Because PMR requires only one symmetry, it can actually be used to compute the RSET in Kerr in two different ways, once using $t$-splitting and once using $\varphi$-splitting. The former primarily relies on the field decomposition in temporal modes $e^{-i \omega t}$, and the latter on (discrete) decomposition in azimuthal modes $e^{i m \varphi}$. Having two independent regularization variants is advantageous as it allows one to test the method's consistency as well as numerical accuracy. Moreover, each splitting variant breaks down in a certain locus, where the norm of the associated Killing field vanishes. This happens to $t$-splitting at the ergosphere boundary, and to $\varphi$-splitting at the polar axis. In reality, the splitting variant becomes problematic also in some neighborhood of that singular locus. Using the two variants allows one to compute the RSET almost everywhere outside the $\mathrm{BH}$.

In this Letter we present the results obtained (from both $t$-splitting and $\varphi$-splitting) for $\left\langle\phi^{2}\right\rangle_{r e n}$ and $\left\langle T_{\alpha \beta}\right\rangle_{r e n}$ in Kerr background, for a minimally-coupled massless scalar field in Unruh state [20] - the quantum state representing an evaporating $\mathrm{BH}$.

Kerr metric and modes computation.- The Kerr metric in Boyer-Lindquist coordinates is

$$
\begin{gathered}
d s^{2}=-\frac{\Delta}{\Sigma}\left(d t-a \sin ^{2} \theta d \varphi\right)^{2}+\frac{\Sigma}{\Delta} d r^{2}+\Sigma d \theta^{2} \\
+\frac{\sin ^{2} \theta}{\Sigma}\left[\left(r^{2}+a^{2}\right) d \varphi-a d t\right]^{2}
\end{gathered}
$$

where $\Delta \equiv r^{2}-2 M r+a^{2}$ and $\Sigma \equiv r^{2}+a^{2} \cos ^{2} \theta, M$ is the BH mass and $a$ its angular momentum per unit mass. We have chosen to work here on the case $a=0.7 \mathrm{M}$, which is strongly motivated by the $\mathrm{BH}$ merger outcomes in the two recent LIGO detections [11]. The field modes were constructed according to the boundary conditions formulated by Ottewill and Winstanley [12. The computation was done by solving the spin-0 Teukolsky equation using a numerical implementation [21] of the ManoSuzuki-Takasugi (MST) formalism [22, 23]. Modes were computed for $-60 \leq m \leq 60$ and for $\omega$ from zero to $\omega_{\max }=8 M^{-1}$ with uniform spacing of $0.01 M^{-1}$. For each $\omega$ and $m$ the sum over $l$ was preformed until sufficient convergence was achieved. In total, just over 4 million $l m \omega$-modes were used.

Results for $\left\langle\phi^{2}\right\rangle_{\text {ren }}$ in Kerr.- $\quad$ In calculating $\left\langle\phi^{2}\right\rangle_{\text {ren }}$ using $t$-splitting [17], one has to integrate a certain function $F_{\text {reg }}(\omega)$ over $\omega$. This function contains oscillations, originating from "connecting null geodesics" (CNGs), [17 whose wavelengths (in $\omega$ ) are dictated by the length (in $t$ ) of these CNGs. The self-cancellation method introduced in Ref. [17] to eliminate the oscillations requires knowledge of these wavelengths. In the Schwarzschild case we determined them by numerically finding the CNGs. In Kerr, however, it is a bit more difficult to compute the CNGs. We therefore used two alternative techniques. The first was finding the wavelengths from a Fourier transform of $F_{r e g}(\omega)$, and self-canceling the oscillations according to the recipe of [17]. The second was simply to apply a low-pass filter to $F_{\text {reg }}(\omega)$, to eliminate the oscillations. The two techniques produced very similar results.

We also computed $\left\langle\phi^{2}\right\rangle_{\text {ren }}$ using $\varphi$-splitting. This variant, which was used recently 19 for the computation of $\left\langle T_{\alpha \beta}\right\rangle_{r e n}$ in Schwarzschild, will be presented in detail elsewhere [24]. We should mention, briefly, that in $\varphi$ splitting regularization we first sum the $l m \omega$ mode contributions over $l$ (a convergent sum), to obtain the functions $F(\omega ; m)$. Next we regularize the integrals of $F(\omega ; m)$ over $\omega$ (for each $m$ ), and finally we regularize the sum over $m$. Here, too, one finds that $F(\omega ; m)$ exhibits oscillations in $\omega$. In some analogy with the angular-splitting case [18, these oscillations originate from CNGs in a fictitious $2+1$ dimensional spacetime (obtained from the Kerr metric by eliminating the $\varphi$ coordinate). We were able to numerically compute these reduced-dimension CNGs and to obtain the oscillations' wavelengths, which we then used to self-cancel the oscillations. After integrating the (smoothened and regularized) functions $F(\omega ; m)$ over $\omega$, the sum over $m$ is regularized using a discrete Fourier decomposition of the counter-terms, in analogy to the Fourier decomposition of the latter in $t$-splitting [17].

Figure 1 displays the results for $\left\langle\phi^{2}\right\rangle_{\text {ren }}$ versus $\theta$ (for various $r$ values) in Unruh state, for $a / M=0.7$, obtained using both variants: $t$-splitting in solid curves, and $\varphi$-splitting in "+" symbols. Here, and also in all other figures, numerical results are given in units $M=1$ (in addition to $G=c=1$ ). Note that for $\varphi$-splitting we only give results for $\theta \geq 30^{\circ}$ because its accuracy rapidly deteriorates on getting closer to the pole. 37] The agreement between the two variants is better than one part in $10^{3}$ throughout the domain presented. This agreement steadily improves with increasing $\theta$ : At $\theta \geq 50^{\circ}$ it is better than two parts in $10^{5}$, and for $\theta=90^{\circ}$ it is about one part in $10^{6}$. We can independently estimate the accuracy of our $t$-splitting results, it is usually better than one part in $10^{6}$ (throughout $4 M \leq r \leq 10 M$ ). Therefore, throughout the domain shown in Fig. 1 we may associate the disagreement between the two variants with the inaccuracy in $\varphi$-splitting. 38.

Results for RSET in Kerr.- The numerical computation of the RSET is much more challenging. It requires 


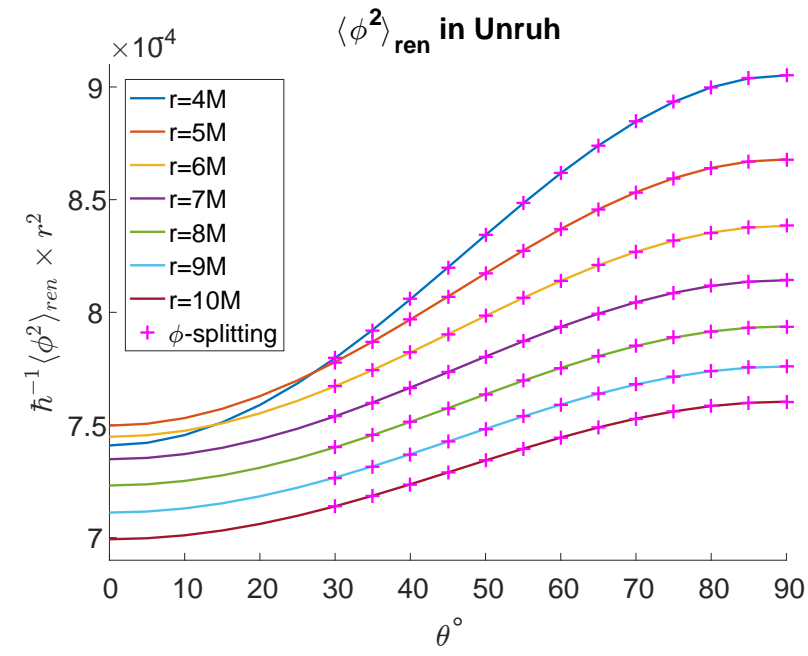

Figure 1: Results for $\left\langle\phi^{2}\right\rangle_{r e n} \times r^{2}$ in Unruh state in Kerr, from both $t$-splitting (solid curves) and $\varphi$-splitting ("+" symbols).

more modes and also higher accuracy for each mode, because the divergence is stronger. As a consequence, our numerical results for $\left\langle T_{\alpha \beta}\right\rangle_{\text {ren }}$ are less accurate than for $\left\langle\phi^{2}\right\rangle_{\text {ren }}$. 39]

We point out that $\left\langle T_{\theta t}\right\rangle_{\text {ren }}$ and $\left\langle T_{\theta \varphi}\right\rangle_{\text {ren }}$ identically vanish (mode by mode) for our massless scalar field. In addition, $\left\langle T_{r t}\right\rangle_{r e n}$ and $\left\langle T_{r \varphi}\right\rangle_{r e n}$ are individuallyconserved components that do not require any regularization. These two components are further addressed below. We shall refer to the remaining six components, which do require regularization, as "nontrivial". Figure 2 displays results for the six nontrivial components of $\left\langle T_{\alpha \beta}\right\rangle_{\text {ren }}$ in Unruh state, as functions of $r$, for two rays: $\theta=90^{\circ}$ and $\theta=0^{\circ}$. In the latter $\varphi$-splitting is invalid, hence only $t$ splitting results are shown. At $\theta=90^{\circ}$ we provide results from both $t$-splitting and $\varphi$-splitting.

We estimate the accuracy of the $t$-splitting results to be better than one part in $10^{3}$ (throughout $r \geq 2.5 \mathrm{M}$ ). The disagreement between the two variants at the equator is at worst $\sim 4 \%$ (for $r=2.5 M$ ), but it is usually better than $\sim 1 \%$, it improves with increasing $r$, and at $r=10$ it is about one part in $10^{3}$. Here again, the disagreement between the two variants is predominantly associated to limited accuracy of $\varphi$-splitting.

Figure 3 displays all six nontrivial RSET components as functions of $\theta$ for $r=6 M$. The $t$-splitting results are estimated to be accurate to about one part in $10^{3}$. We also present results from $\varphi$-splitting for $\theta \geq 30^{\circ}$. The disagreement between the two is fairly large (easily visible) at $\theta=30^{\circ}$, but it improves with increasing $\theta$. It is a few percents for $\theta=35^{\circ}$ and reduces to a few parts in $10^{3}$ at the equator.

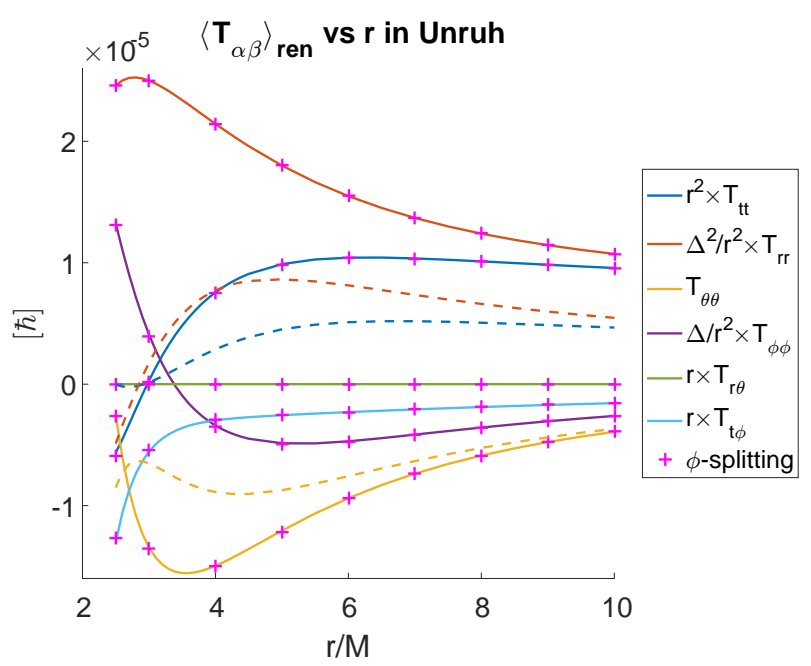

Figure 2: The six nontrivial RSET components as functions of $r$. The solid curves and "+" symbols are results at $\theta=$ $90^{\circ}$ from $t$-splitting and $\varphi$-splitting respectively. The dashed curves are $t$-splitting results at $\theta=0$. Notice that $\left\langle T_{r \theta}\right\rangle_{r e n}$ (green line) vanishes at both the pole and equator, due to obvious symmetry properties. Also, $\left\langle T_{\varphi \varphi}\right\rangle_{\text {ren }}=\left\langle T_{t \varphi}\right\rangle_{\text {ren }}=0$ at the pole.

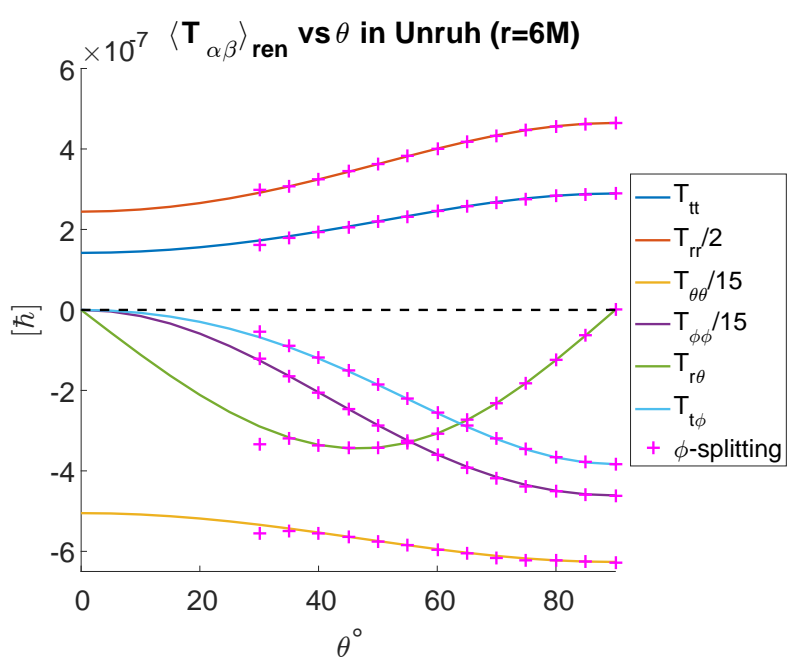

Figure 3: The six nontrivial RSET components at $r=6 M$ as functions of $\theta$. The solid curves and the "+" symbols are results obtained from $t$-splitting and $\varphi$-splitting respectively. The deviations between the two are visible at $\theta=30^{\circ}$.

Energy-momentum conservation and conserved fluxes.- An important consistency check of the computed RSET is energy-momentum conservation 
$\left(\left\langle T^{\alpha \beta}\right\rangle_{\text {ren }}\right)_{; \beta}=0$. In the PMR method one subtracts certain known tensors (derived from Christensen's counter-terms) from the otherwise-divergent mode-sum. We have analytically checked that these tensors are all conserved. This ensures that the resultant RSET is conserved too, because the contribution from the individual modes is guaranteed to be conserved. We have also directly checked, numerically, the conservation of our resultant RSET. 40,

Two components of the conservation equation, $\alpha=t$ and $\alpha=\varphi$, yield especially simple conservation laws:

$$
\left\langle T_{r t}\right\rangle_{r e n}=-K(\theta) / \Delta, \quad\left\langle T_{r \varphi}\right\rangle_{r e n}=L(\theta) / \Delta .
$$

The $r$-independent quantities $K(\theta)$ and $L(\theta)$ respectively represent the outgoing energy and angularmomentum flux densities (multiplied by $r^{2}$ ), as measured by far observers placed at various $\theta$ values. Note that these fluxes do not vanish even in Boulware state, due to the Unruh-Starobinsky effect 25, 26. Our results for $K(\theta)$ and $L(\theta)$ are displayed in Fig. 4 for both Unruh and Boulware states. Although the computation of $K(\theta)$ and $L(\theta)$ does not require regularization, to the best of our knowledge it is the first time they are presented for a scalar field (results for electromagnetic field are given in [13).

The integrals of $K(\theta)$ and $L(\theta)$ over the entire two-sphere yield the total energy flux $f$ and angularmomentum flux $g$ emitted to infinity. In Unruh state we obtain $f=7.166 \cdot 10^{-5} \hbar / M^{2}$ and $g=1.8116 \cdot 10^{-4} \hbar / M$. Of course these quantities can also be directly computed using Hawking's original method [1, which only requires numerical computation of the reflection and transmission coefficients. This calculation (for a scalar field in Kerr) was done by Taylor, Chambers and Hiscock 27]. Our results agree with their computation to better than $1 \%$, which is their declared accuracy. To examine it more carefully we have repeated their Hawking-radiation calculation, using MST method [21] for the reflection and transmission coefficients. We found agreement better than one part in $10^{4}$ with the above mentioned results for $f$ and $g$ (obtained from integrating $K, L$ ).

For Boulware state we obtain the integrated fluxes $f^{B}=1.265 \cdot 10^{-6} \hbar / M^{2}$ and $g^{B}=1.2187 \cdot 10^{-5} \hbar / M$, which express the Unruh-Starobinsky effect.

Discussion.- We employed our PMR method to compute $\left\langle\phi^{2}\right\rangle_{\text {ren }}$ and $\left\langle T_{\alpha \beta}\right\rangle_{\text {ren }}$ in a spinning $\mathrm{BH}$, for a minimally-coupled massless scalar field, in both Unruh and Boulware states. For brevity we mostly presented results for the more realistic Unruh state, which represents physical evaporating BHs. In addition, for Boulware state we displayed the fluxes of energy and angular momentum to infinity (the Unruh-Starobinsky effect).

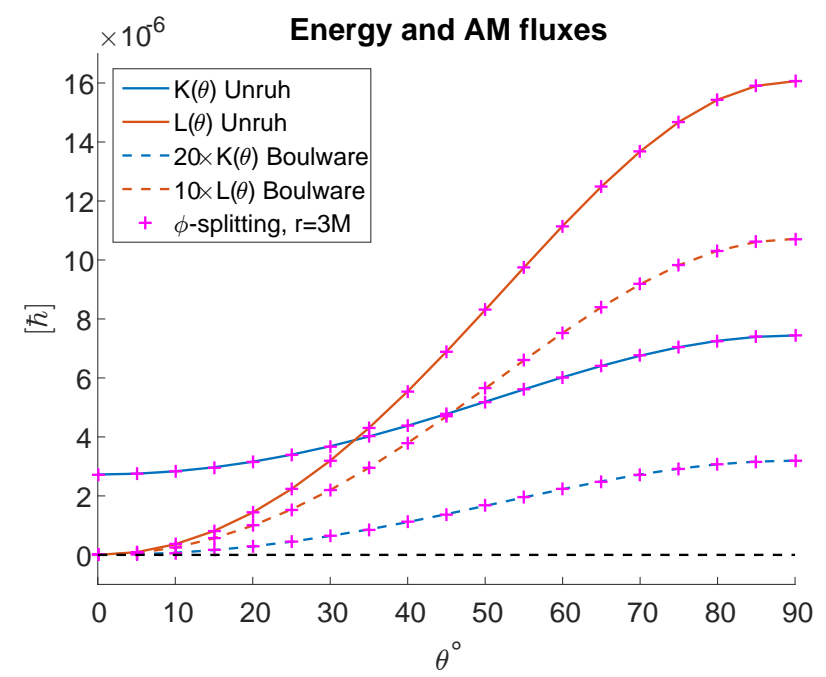

Figure 4: The energy flux $K(\theta)$ and angular-momentum flux $L(\theta)$. The solid curves are results in Unruh state, and the dashed curves are in Boulware state - both obtained using $t$-splitting at $r=10 M$. The "+" symbols are results obtained using $\varphi$-splitting at $r=3 M$ (also confirming the $r$-independence of $K$ and $L$ ).

The regularization was done once using the $t$-splitting variant, exploiting Kerr's stationarity, and once using the $\varphi$-splitting variant, exploiting its axial symmetry, with good agreement between the two variants in the regime where they both function properly.

The usage of the two variants enables us to cross-check our results, and it also helps assessing the numerical accuracy. In the domain of $r$ and $\theta$ for which we have presented results, $t$-splitting always provided more accurate results (This would change on approaching the ergosphere boundary, where the temporal Killing field becomes null). Note that the $\varphi$-splitting variant is more challenging, as it requires two regularizations: the integral over $\omega$ (as in $t$-splitting), and also the sum over $m$. One would of course expect $\varphi$-splitting to fail at the polar axis, as the azimuthal Killing field vanishes. We were a bit surprised to see that $\varphi$-splitting starts to severely deteriorate fairly far from the pole: say, for $r=6 M$, at $\theta \lesssim 15^{\circ}$ for $\left\langle\phi^{2}\right\rangle_{r e n}$ and at $\theta \lesssim 35^{\circ}$ for $\left\langle T_{\alpha \beta}\right\rangle_{r e n}$. The situation improves with increasing $\omega_{\max }$.

One of the ultimate goals of the $\varphi$-splitting variant is to allow investigating back-reaction effects in timedependent, spinning, evaporating BHs. We see that in its present form $\varphi$-splitting is not yet capable of achieving this goal, primarily due to the wide domain of inapplicability around the pole. We do hope to improve this situation.

Besides extending the computation to other $a / M$ values, and besides improving the $\varphi$-splitting variant, we see several obvious extensions of this work. The first is to compute the RSET inside the ergosphere and also 
inside the horizon. This is important for understanding how semiclassical effects would modify the internal structure of spinning BHs. The second is to repeat our computation for other fields, e.g. the electromagnetic field. Another possible use of our PMR method is the RSET computation in the background metric of rapidlyrotating relativistic stars (e.g. a neutron star).

We also hope that the ability to compute the RSET (and its corresponding back-reaction) in a spinning $\mathrm{BH}$ will open the door for computing semiclassical corrections to various physical phenomena. A possible example is corrections to quasi-normal modes of evaporating spinning BHs 28, 29]; Another potential use for the RSET is in the context of the Kerr/CFT corresponds [31-34.

Full detail of this analysis of $\left\langle\phi^{2}\right\rangle_{\text {ren }}$ and $\left\langle T_{\alpha \beta}\right\rangle_{\text {ren }}$ in a Kerr BH will be presented elsewhere.

Acknowledgment.- We are grateful to Leor Barack for many fruitful discussions, and to Marc Casals for his helpful advice. This research was supported by the Asher Fund for Space Research at the Technion. MvdM was supported by the European Research Council under the European Union's Seventh Framework Programme (FP7/2007-2013) ERC grant agreement no. 304978. The numerical results in this paper were obtained using the IRIDIS High Performance Computing Facility at the University of Southampton.

[1] S. W. Hawking, Commun. Math. Phys. 43,199 (1975).

[2] B. S. DeWitt, Dynamical Theory of Groups and Fields (Gordon and Breach, New York, 1965).

[3] S. M. Christensen, Phys. Rev. D 14, 2490 (1976).

[4] P. Candelas and K. W. Howard, Phys. Rev. D 29, 1618 (1984).

[5] K. W. Howard, Phys. Rev. D 30, 2532 (1984).

[6] P. R. Anderson, Phys. Rev. D 41, 1152 (1990).

[7] P. R. Anderson, W. A. Hiscock, and D. A. Samuel, Phys. Rev. D 51, 4337 (1995).

[8] P. Taylor and C. Breen, arXiv:1609.08166.

[9] T. Elster, Phys. Lett. 94 A, 205 (1983).

[10] B. P. Jensen, J. G. McLaughlin, and A. C. Ottewill, Phys. Rev. D 43, 4142 (1991).

[11] B. P. Abbott et al. (LIGO Scientific Collaboration and Virgo Collaboration), arXiv:1606.04856.

[12] A. C. Ottewill and E. Winstanley, Phys. Rev. D 62, 084018 (2000).
[13] M. Casals and A. C. Ottewill, Phys. Rev. D 71, 124016 (2005).

[14] G. Duffy and A. C. Ottewill, Phys. Rev. D 77, 024007 (2008).

[15] M.Casals, A. Fabbri, C. Martínez and J. Zanelli, arXiv:1608.05366.

[16] H. R. C. Ferreira, Int. J. Mod. Phys. D 24, 1542007 (2015).

[17] A. Levi and A. Ori, Phys. Rev. D 91, 104028 (2015).

[18] A. Levi and A. Ori, Phys. Rev. D 94, 044054 (2016).

[19] A. Levi and A. Ori, arXiv:1608.03806.

[20] W. G. Unruh, Phys. Rev. D 14, 870 (1976).

[21] M. van de Meent, in preparation.

[22] S. Mano, H. Suzuki, and E. Takasugi, Prog. Theor. Phys. 95,1079-1096, (1996).

[23] S. Mano and E. Takasugi, Prog. Theor. Phys. 97, 213$232,(1997)$

[24] A. Levi and A. Ori, in preparation.

[25] A. A. Starobinskii, Sov. Phys. JETP 37 28, (1973).

[26] W. G. Unruh, Phys. Rev. D 103194 (1974).

[27] B. E. Taylor, C. M. Chambers, and W. A. Hiscock, Phys. Rev. D 58, 044012 (1998).

[28] O. P. F. Piedra and J. de Oliveira, Class. Quantum Grav. 28085003 (2011).

[29] A. Belokogne and A. Folacci, Phys. Rev. D 90, 044045 (2014).

[30] W. H. Press and S. A. Teukolsky, Nature 238, 211 - 212 (1972).

[31] M. G. T. Hartman, W. Song, and A. Strominger, Phys. Rev. D 80, 124008 (2009).

[32] A. Castro, A. Maloney, and A. Strominger, Phys. Rev. D 82, 024008 (2010).

[33] I. Agullo, J. Navarro-Salas, G. J. Olmo, and L. Parker, Phys. Rev. Lett. 105, 211305 (2010).

[34] I. Bredberg, T. Hartman, W. Song and A. Strominger, J. High Energ. Phys. 1004:019 (2010).

[35] A. L. Matacz, P. C. W. Davies and A. C. Ottewill, Phys. Rev. D 47, 1557 (1993).

[36] An interesting new method (still for spherical static backgrounds) was recently proposed by Taylor and Breen [8].

[37] In fact the relevant parameter is $r \sin \theta$.

[38] This situation is reversed in the neighborhood of $r \approx$ $2 M, \theta>1$ (not included in Fig. 1).

[39] The aforementioned oscillations in $\omega$ show up in the RSET computation too, and we handle them (in both variants) just as we did in the calculation of $\left\langle\phi^{2}\right\rangle_{r e n}$.

[40] Due to the basic consistency of the different variants it is sufficient to verify energy-momentum conservation in one of the variants. Here we use $t$-splitting for this purpose, because it is technically simpler (and numerically more accurate). 